\title{
Synthesis and Microstructural Characterization of Fe-Cu Nanoparticles Growth by Chemical Reduction
}

\author{
R. Morales-Luckie, ${ }^{* * *}$ V. Sánchez-Mendieta,* R. López-Castañares ${ }^{*}$ and J. Arenas-Alatorre ${ }^{* *}$
}

*Laboratorio de Investigación y Desarrollo de Materiales Avanzados, Facultad de Química, Universidad Autónoma del Estado de México, Apdo. Postal A-20, Toluca, México C.P. 50120.

**Departamento de Materia Condensada, Instituto de Física, UNAM, Apdo. Postal 20-364 México 01000 D.F. Corresponding author jarenas@fisica.unam.mx.

In the last years has been increasing interest in artificially grown metastable alloys from insoluble binary metallic systems as they offer a variety on new properties and applications [1]. An example of these systems is the $\mathrm{Fe}-\mathrm{Cu}$, which shows a complete immiscibility in the solid state under equilibrium conditions [2] and many works has been reported related to mechanical alloying [3], thin films and magnetic properties of this system [4].

In this work we explain the study of the $\mathrm{Fe}-\mathrm{Cu}$ nanoparticles system growth by chemical synthesis and characterized by High Resolution Electron Microscopy (HREM), X-Ray Difraction (XRD) and using, in addition, image digitalization techniques. The $\mathrm{Fe}-\mathrm{Cu}$ nanoparticles were grown using $\mathrm{FeSO}_{4} \cdot 7 \mathrm{H}_{2} \mathrm{O}(\mathrm{J} . \mathrm{T}$. Baker), $\mathrm{CuSO}_{4} \cdot 5 \mathrm{H}_{2} \mathrm{O}$ (J.T. Baker) and $\mathrm{NaBH}_{4}$ (Aldrich). Synthesis of nano-particles was carried out in presence of air, at room temperature and atmospheric pressure conditions. Solutions of $5 \mathrm{mM}$ of both metal salts, and of $10 \mathrm{mM}$ of reducing agent, were prepared utilizing de-ionized water; then, solutions of both metal salts were mixed in order to have molar ratios of 50-50\%at. (Fe- $\mathrm{Cu}$ ) and 30-70 \% at., (Fe$\mathrm{Cu}$ ), respectively. These solutions were stirred during three minutes, after that, $\mathrm{NaBH}_{4}$ solution is added, at once, to the mixture. A dark precipitate appears, which is filtered, and washed thoroughly, first with de-ionized water and then with acetone, this last step was done in a glove box having a $\mathrm{N}_{2}$ atmosphere. Micrographs were obtained with a TEM JEM-2010F and XRD were acquired with a Siemens D5000 diffractometer, operated to $35 \mathrm{KeV}$, at room temperature with a $\mathrm{Cu} \mathrm{K} \alpha$ radiation.

The XRD results show that in the sample $\mathrm{Cu}_{50} \mathrm{Fe}_{50}$, the reflections of the phase of Fe disappear and only those corresponding to $\mathrm{Cu}$ are observed. On the other hand, in the sample $\mathrm{Cu}_{70} \mathrm{Fe}_{30}, \mathrm{Cu}$ and $\mathrm{Cu}_{2} \mathrm{O}$ were identified but $\mathrm{Fe}$ and their oxides were not recognized. HREM image showed core-shell nanoparticles (figure 1), with crystalline core and amorphous shell. In figure 2 a a nanoparticle with many crystalline defects in the core and an amorphous shell is observed. A third shell is observed with interplanar distances near to graphite. In figure $2 b$ the FFT analyses of the core indicate a FCC structure with interplanar distances corresponding to $\mathrm{Cu}$. Crystalline areas, which might be attributable to Fe, are embedded in the amorphous shell, as shown in figure $2 \mathrm{~b}$. This work is a contribution to the understanding of the metaestable alloy $\mathrm{Fe}-\mathrm{Cu}$ as a nanosystem.

\section{References}

[1].M.K. Roy and H.C. Verma, J.Magn.Magn. Mater. 270(2004)186.

[2] T.B. Massalski (ed), Binary Alloy Phase Diagram, ASM, Metals Park, hio, 1986.

[3] J.Z. Jiang et al., Appl. Phys. Lett. 63(20) (1993) 2768.

[4] N.H. Duc et al., J. Phys.: Condens. Matter 14(2002) 6657.

[5] This work was supported by UNAM trough grant PAPIIT- IX118004 and UAEM 1950/2004. We are grateful to L. Rendón, R. Hernández and Samuel Tehuacanero for technical help 

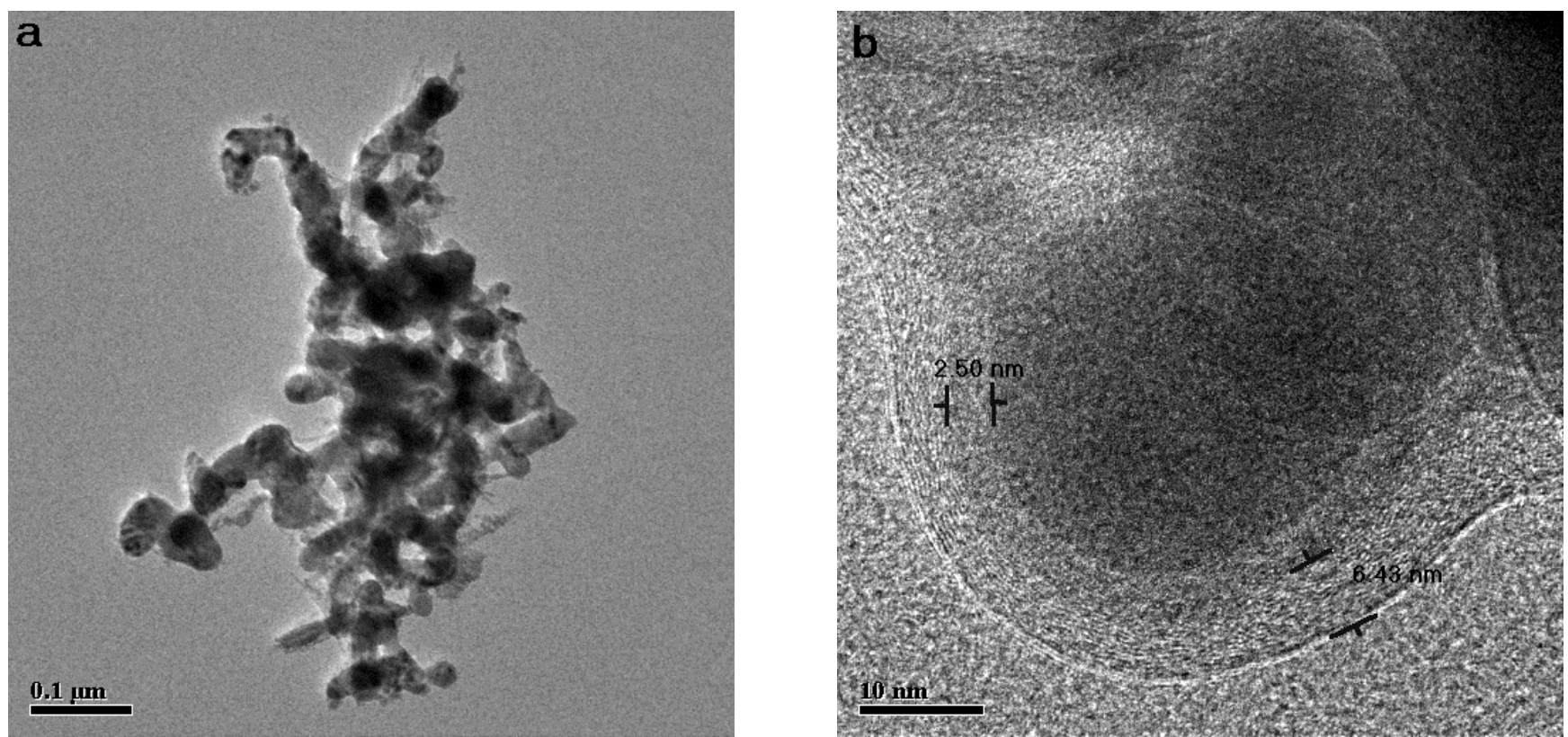

Figure 1.-a) TEM image of $\mathrm{Cu}_{50} \mathrm{Fe}_{50}$ where particles with high contrast and smaller that $450 \mathrm{~nm}$ are observed. These particles are surrounded of zones of irregular morphology and low contrast. b) HREM image of a core shell nanoparticle where is observed an amorphous shell of $2.50 \mathrm{~nm}$ of thickness. The external shell of $6.48 \mathrm{~nm}$ of thicknes has interplanar distances of $0.35 \mathrm{~nm}$.
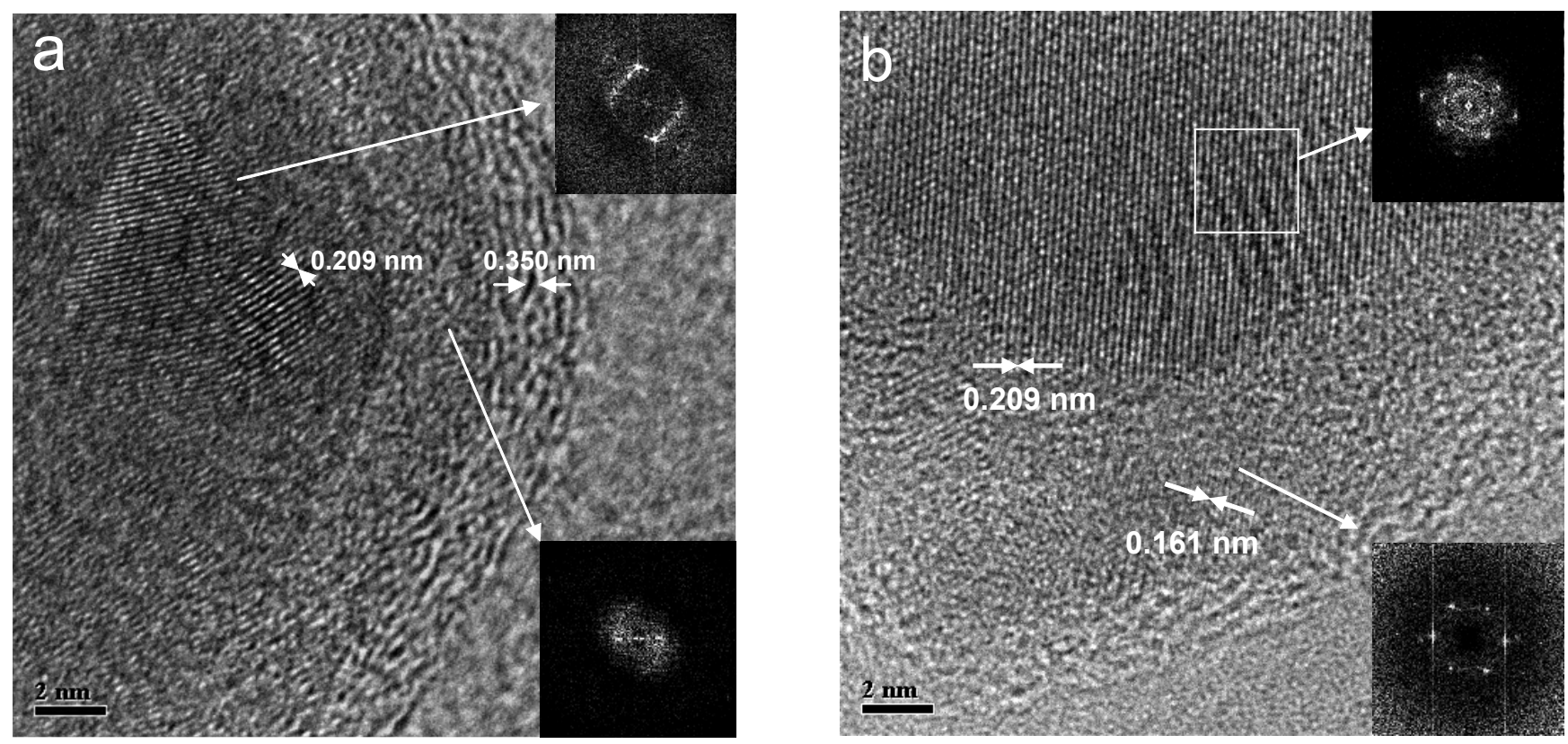

Figure 2.- a) Nanoparticle of the sample $\mathrm{Cu}_{70} \mathrm{Fe}_{30}$. The core showing many crystalline defects, the first shell is amorphous and the interplanar distance of the second shell is near to graphite. b) Nanoparticle with interplanar distances of the core corresponding to $\mathrm{Cu}$ (FCC structure), and a crystalline area embedded in the amorphous shell, with interplanar distance of $0.161 \mathrm{~nm}$. 